\title{
Enhanced Effect of Mesoporous Silica on Base-Catalyzed Aldol Reaction
}

\author{
Yoshihiro Kubota, ${ }^{*}$ Kunio Goto, Shintaro Miyata, Yasutomo Goto ${ }^{\dagger}$ Yoshiaki Fukushima, $^{\dagger}$ and Yoshihiro Sugi* \\ Department of Materials Science and Technology, Faculty of Engineering, Gifu University, Gifu 501-1193
}

${ }^{\dagger}$ Toyota Central R\&D Labs., Inc., Nagakute, Aichi 480-1192

(Received November 22, 2002; CL-020999)

Aldol reaction of 4-nitrobenzaldehyde with acetone gives 4(4-nitrophenyl)-4-hydroxy-2-butanone in high yield in the presence of both secondary amine and mesoporous silica, whereas the yield is low in the absence of the mesoporous silica.

High-silica, periodical mesoporous materials are recognized to be useful as catalysts and sorption materials because they possesses hydrophobic pores with uniform size, large surface area, large adsorption capacity, and high thermal stability. ${ }^{1}$ This class of materials is becoming important as the catalyst or catalyst support for the synthesis of fine chemicals, in which the reaction conditions consist of low temperature and liquid phase. Recently, examples of solid base-catalyzed $\mathrm{C}-\mathrm{C}$ bond forming reactions, such as Knoevenagel condensation, ${ }^{2-4}$ aldol condensation, ${ }^{3,5}$ and Michael addition, ${ }^{6,7}$ have been disclosed. In this work, we focused on the aldol reaction of aromatic aldehyde with ketone. As for the solid base catalysis, a few examples of this reaction have been reported so far; in which the catalysts are amine- or quaternary ammonium-immobilized mesoporous materials ${ }^{3,8}$ or hydrotalcite, ${ }^{9}$ and the major products are "dehydrated" or further reacted products rather than more desired aldol adducts $(\beta$ hydroxyketones). When using the amine-immobilized mesoporous silicas as basic catalysts, the real effects of amine site and silica support have not been well investigated. In the present work, we examined the effect of amine itself as well as the silicate such as FSM- $16^{10}$ and MCM- $41^{11}$ as additive on the reaction, and found a prominent conjugate effect of the silicate. The catalytic performance of amine-immobilized silicate in the same reaction was investigated as well.

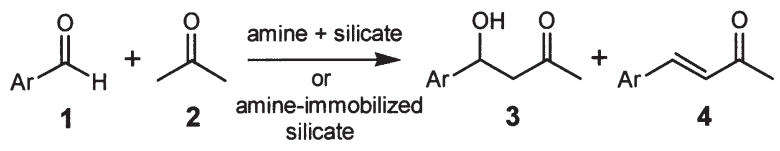

Mesoporous silicas were synthesized by known procedures ${ }^{12,13}$ using hexadecylammonium cation as structuredirecting agent. Periodical mesoporous structures were confirmed by X-ray diffraction and $\mathrm{N}_{2}$ adsorption measurements. BET surface areas $\left(S_{\mathrm{BET}}\right) /$ average pore diameters (BJH method) for FSM-16 and MCM-41 were $1060 \mathrm{~m}^{2} \mathrm{~g}^{-1} / 2.7 \mathrm{~nm}$ and $1010 \mathrm{~m}^{2} \mathrm{~g}^{-1} /$ $2.9 \mathrm{~nm}$, respectively. The typical reaction in the presence of amine and solid additive was carried out as follows: a solution of 4-nitrobenzaldehyde (1a, $1.0 \mathrm{mmol})$ in acetone $(\mathbf{2}, 5 \mathrm{ml})$ was maintained at $30^{\circ} \mathrm{C}$ with stirring. To this solution, piperazine $(0.1 \mathrm{mmol})$ and FSM-16 (120 mg) were added successively, and the whole mixture was stirred at the same temperature for $6 \mathrm{~h}$. The reaction mixture was filtered to remove solid additive and the residue was washed with benzene $(100 \mathrm{ml})$. The combined filtrate was washed with chilled $10 \%$ citric acid solution (in order to avoid the further catalysis by the remaining amine), saturated
$\mathrm{NaHCO}_{3}$ solution followed by brine, and then dried over $\mathrm{Na}_{2} \mathrm{SO}_{4} \cdot{ }^{14}$ Filtration and evaporation gave yellow crude solid, which was purified by column chromatography on silica gel (eluent: hexane-ethyl acetate) to give $1.1 \mathrm{mg}(0.7 \%)$ of $\mathbf{1 a}$, $191.1 \mathrm{mg}$ (90.8\%) of 4-(4-nitrophenyl)-4-hydroxy-2-butanone (3a), and $7.0 \mathrm{mg}(3.6 \%)$ of (E)-4-(4-nitrophenyl)-3-buten-2-one (4a).

Table 1 shows the effect of secondary amine on the reaction of $\mathbf{1 a}$ with $\mathbf{2}$. The activities were generally low and the yields of major product 3a were not exceeding 5\% (Entries 1, 6 and 8). Surprisingly, the product yield enormously increased by addition of FSM-16 into the reaction mixture containing $10 \mathrm{~mol} \%$ of piperidine, piperazine, or dipropylamine (Entries 2, 7 and 9). Sterically hindered amines showed no activity with or without FSM-16 (Entries 10-13). Effect of MCM-41 was almost the same as that of FSM-16, whereas amorphous silica (Davison \#57; $S_{\mathrm{BET}}=370 \mathrm{~m}^{2} \mathrm{~g}^{-1}$ ) had weaker effect (Entries 3 and 4 ). The silicates in the absence of amine was totally inert (Entry 5), indicating that the high activity is realized only when the reaction is carried out in the presence of both amine and silicate additive. Primary amine (propylamine, hexylamine, benzylamine, and cyclohexylamine) showed only low activity in the reaction of $\mathbf{1 a}$ with 2 (conversion $<20 \%$ ), and in each case the activity did not change even when FSM-16 was added. The use of tertiary amine such as triethylamine, tributylamine, diisopropylethylamine, 1,4diazabicyclo[2.2.2] octane (DABCO), and pyridine, either with or without FSM-16, did not give any product and only 1a was recovered quantitatively. Thus the activity of this catalytic system is obviously dependent on the type of amines besides the presence or absence of silicate additive. The order of activity (secondary $>$ primary $>$ tertiary) is quite reasonable in light of the proposed meshanism via enamine formation. ${ }^{15}$ We could thus observe the

Table 1. Aldol reaction of 4-nitrobenzaldehyde (1a) with acetone (2) catalyzed by secondary amine with or without additive ${ }^{\mathrm{a}}$

\begin{tabular}{|c|c|c|c|c|c|}
\hline \multirow{2}{*}{ Entry } & \multirow{2}{*}{ Base $^{b}$} & \multirow{2}{*}{ Additive } & \multirow{2}{*}{$\begin{array}{c}\text { Conv. } \\
1 \%\end{array}$} & \multicolumn{2}{|c|}{ Isolated yield/\% } \\
\hline & & & & $3 \mathbf{a}$ & $4 a$ \\
\hline 1 & Piperidine & - & 7 & 3 & 0 \\
\hline 2 & Piperidine & FSM-16 & 98 & 80 & 5 \\
\hline 3 & Piperidine & MCM-41 & 97 & 79 & 6 \\
\hline 4 & Piperidine & $\mathrm{SiO}_{2}$ & 59 & 47 & 2 \\
\hline 5 & - & FSM-16 & 0 & 0 & 0 \\
\hline 6 & Piperazine & - & 11 & 5 & 1 \\
\hline 7 & Piperazine & FSM-16 & 99 & 91 & 4 \\
\hline 8 & $n-\mathrm{Pr}_{2} \mathrm{NH}$ & - & 6 & 2 & 0 \\
\hline 9 & $n-\mathrm{Pr}_{2} \mathrm{NH}$ & FSM-16 & 79 & 48 & $5^{c}$ \\
\hline 10 & $i-\mathrm{Pr}_{2} \mathrm{NH}$ & - & 0 & 0 & 0 \\
\hline 11 & $i-\mathrm{Pr}_{2} \mathrm{NH}$ & FSM-16 & 0 & 0 & 0 \\
\hline 12 & 2,6-Lutidine & - & 0 & 0 & 0 \\
\hline 13 & 2,6-Lutidine & FSM-16 & 0 & 0 & 0 \\
\hline
\end{tabular}

${ }^{\mathrm{a}}$ The reaction was carried out at $30^{\circ} \mathrm{C}$ as described in the text. ${ }^{\mathrm{b}} 10 \mathrm{~mol} \%$ of each amine was used. ${ }^{\mathrm{c}} \mathrm{A} 18 \%$ of 2,6-di(4-nitrophenyl)tetrahydropyran-4-one was also obtained as a further reacted product. 
efficiency of amine moieties and silicates separately. The high performance of piperidine-type secondary amine in combination with mesoporous silica is valuable in view of the simplicity of reaction procedure.

As shown in Table 2, lower reactivities and selectivities were observed for aromatic aldehydes other than 1a. However, the positive effect of FSM-16 was obvious. The addition of FSM-16 increased not only conversion but also the selectivity for $\beta$ hydroxyketone 3 . In this catalytic system, aldehyde having electron-withdrawing group seems to be preferable for the selective formation of $\mathbf{3}$.

Table 2. Aldol reaction of $\mathbf{1 b}-\mathbf{e}$ with $\mathbf{2}$ catalyzed by piperidine with or without additive ${ }^{\mathrm{a}}$

\begin{tabular}{|c|c|c|c|c|c|c|c|}
\hline \multirow{2}{*}{ Entry } & \multirow{2}{*}{$\mathrm{Ar}$} & \multirow{2}{*}{1} & \multirow{2}{*}{ Additive } & \multicolumn{4}{|c|}{ Temp. Conv. Yield $/ \%$} \\
\hline & & & & $1{ }^{\circ} \mathrm{C}$ & $1 \%$ & 3 & 4 \\
\hline 1 & 4-Chlorophenyl & 1b & - & 30 & 7 & 2 & 3 \\
\hline 2 & 4-Chlorophenyl & $1 b$ & FSM-16 & 30 & 54 & 26 & 11 \\
\hline 3 & Phenyl & 1c & - & 30 & 5 & 1 & 3 \\
\hline 4 & Phenyl & 1c & FSM-16 & 30 & 36 & 20 & 15 \\
\hline 5 & 4-Methylphenyl & 1d & - & 30 & 8 & 1 & 6 \\
\hline 6 & 4-Methylphenyl & 1d & FSM-16 & 30 & 19 & 6 & 13 \\
\hline 7 & 3,4,5-Trimethoxyphenyl & 1e & - & 60 & 0 & 0 & 0 \\
\hline 8 & 3,4,5-Trimethoxyphenyl & 1e & FSM-16 & 60 & 49 & 17 & 19 \\
\hline
\end{tabular}

${ }^{\mathrm{a}}$ The reaction was carried out as described in the text. ${ }^{\mathrm{b}}$ Isolated yields.

We also investigated the effect of amine-immobilized silicates (Figure 1). 3-Aminopropyl (AP)-, 3-piperazinopropyl (PzP)-, $N$-methylaminopropyl (MAP)-, and $N, N$-dimethylaminopropyl (DMAP)-functionalized materials were used. Immobilization of basic site on silicate was carried out according to the reported procedure with slight modification. ${ }^{16}$ Among the immobilized amines tested, secondary amine showed the highest activity. This is the same tendency as described above, suggesting that enamine may be the key intermediate. The particularly high activity shown by MAP-FSM-16 (yield of 3a and $\mathbf{4 a}$ after $1 \mathrm{~h}$ were $86 \%$ and $7 \%$, respectively) is probably due to the facile formation of enamine. The combined yield of $\mathbf{3 a}$ and $\mathbf{4 a}$ was only $16 \%$ when the reaction was carried out in the presence of $\mathrm{PrNH}_{2}(19 \mathrm{~mol} \%)$ + FSM-16, whereas it increased significantly ( $>50 \%$ as shown in Figure 1) when the reaction was catalyzed by AP-FSM-16 ( $14 \mathrm{~mol} \%$ of active site). This means that "immobilization" enhances the reactivity much better than "simple physical mixing". The order of activity (AP-MCM-41, AP-FSM$\left.16>\mathrm{AP}-\mathrm{SiO}_{2}\right)$ indicates again that the periodical structure is advantageous than amorphous. This tendency was consistent with the results given in Table 1, Entries 2-4. Moreover, the same trend was observed in the case of 1,4-addition of aldehydes to vinylketones. ${ }^{17}$

The prominent effect of the silicate support may be partly due to the increase in the reactant concentration on the surface of silicate. ${ }^{17}$ However, we believe that the concomitant acidic moiety along with the basic site is playing some important roles in this organic transformation. Very recently, asymmetric reaction of $\mathbf{1 a}$ with $\mathbf{2}$ catalyzed by L-proline analogue ${ }^{15}$ or the combination of chiral diamine and protonic acid ${ }^{18}$ was successful to give $(R)-3 \mathbf{a}$. These results revealed that a synergistic effect of base with acid is important for this kind of reaction. Proton provided from the Brønsted acid is considered to be participating in the proposed transition state. ${ }^{15}$ Similarly, in our reaction system silanols on the silicate wall

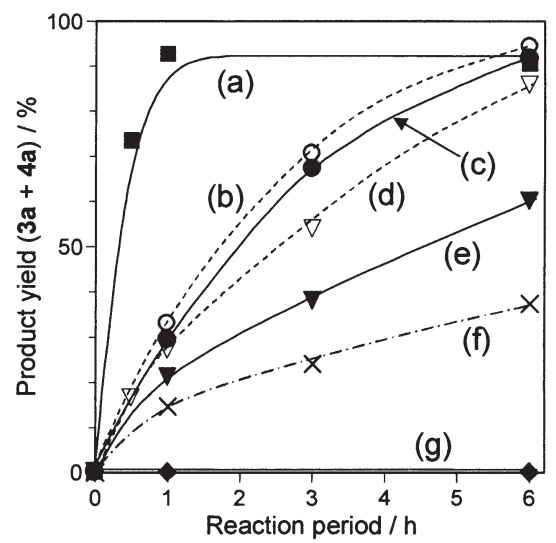

Figure 1. Time-course of the reaction of 1 a with 2 catalyzed by various amineimmobilized silicates: (a) MAP-FSM-16, (b) PzP-MCM-41, (c) PzP-FSM-16, (d) AP-MCM-41, (e) AP-FSM-16, (f) AP-SiO ${ }_{2}$, and (g) DMAP-FSM-16. $120 \mathrm{mg}$ of each catalyst per milimole of $\mathbf{1 a}$ was used. Each catalyst contains $1.30 \pm 0.05 \mathrm{mmol} \mathrm{g}^{-1}$ of effective nitrogen on the basis of elemental analysis.

could be assisting the increase in reactivity in the same manner as suggested in cited Ref. 4 . The effect should be dependent on the acidic property, and the difference in the promoting effect between mesoporous and amorphous silicas might be due to the difference in acidity of silanols inside them. ${ }^{19}$

In summary, we demonstrated that the catalytic performance of a piperidine-type secondary amine for the aldol reaction is significantly improved by addition of a mesoporous silica to give the aldol adduct in relatively high selectivity. Although immobilization is more advantageous towards eco-benignity, this will provide another simple and efficient way to obtain $\beta$-hydroxyketones by aldol reaction. Additionally, this is another clear example of the promoting effect of silicate support. ${ }^{4,17}$

References and Notes

1 J. Y. Ying, C. P. Mehnert, and M. S. Wong, Angew. Chem., Int. Ed., 38, 56 (1999).

2 D. J. Macquarrie, Green Chem., 1999, 195.

3 I. Rodoriguez, Chem. Commun., 1999, 1005

4 Y. Inaki, Y. Kajita, H. Yoshida, K. Ito, and T. Hattori, Chem. Commun., 2001, 2358.

5 K. R. Kloetstra and H. van Bekkum, J. Chem. Soc., Chem. Commun., 1995, 1005 .

6 H. Hattori, Chem. Rev, 95, 537 (1993).

7 Y. Kubota, Y. Nishizaki, H. Ikeya, J. Nagaya, and Y. Sugi, Stud. Surf. Sci. Catal., 141, 553 (2002).

8 B. M. Choudary, M. L. Kantam, P. Sreekanth, T. Bandopadhyay, F. Figueras, and A. Tuel, J. Mol. Catal. A: Chem., 142, 361 (1999).

9 M. L. Kantam, B. M. Choudary, Ch. V. Reddy, K. K. Rao, and F. Figueras, Chem. Commun., 1998, 1033.

10 S. Inagaki, Y. Fukushima, and K. Kuroda, J. Chem. Soc., Chem. Commun., 1993, 680 .

11 C. T. Kresge, M. E. Leonowicz, W. J. Roth, J. C. Vartuli, and J. S. Beck, Nature, 359, 710 (1992)

12 S. Inagaki, A. Koiwai, N. Suzuki, Y. Fukushima, and K. Kuroda, Bull. Chem. Soc. Jpn., 69, 1449 (1996).

13 C.-Y. Chen, H.-X. Li, and M. E. Davis, Microporous Mater., 2, 17 (1993).

14 The washing procedure can be skipped for preparative purpose.

15 K. Sakthivel, W. Notz, T. Bui, and C. F. Barbas, III, J. Am. Chem. Soc., 123, 5260 (2001).

16 E. Angeletti, C. Canepa, G. Martinetti, and P. Venturello, J. Chem. Soc., Perkin Trans. 1, 1989, 105.

17 K. Shimizu, H. Suzuki, E. Hayashi, T. Kodama, Y. Tsuchiya, H. Hagiwara, and Y. Kitayama, Chem. Commun., 2002, 1068.

18 M. Nakadai, S. Saito, and H. Yamamoto, Tetrahedron, 58, 8167 (2002).

19 T. Yamamoto, T. Tanaka, T. Funabiki, and S. Yoshida, J. Phys. Chem. B, 102, 5830 (1998). 